\title{
Ultrasound-Promoted Synthesis, Structural Characterization and in vitro Antimicrobial Activity of New 5-Aryl-3-(2-hydroxyphenyl)-4,5-dihydro- $1 H$-pyrazole-1-carboximidamides
}

\author{
Danilo Y. de Albuquerque, ${ }^{a}$ Andressa C. Damim, ${ }^{b}$ Eliandro Faoro, ${ }^{a}$ \\ Gleison A. Casagrande, ${ }^{a}$ Davi F. Back, ${ }^{c}$ Sidnei Moura, ${ }^{d}$ Claudio M. P. de Pereira, ${ }^{e}$ \\ Kelly M. P. de Oliveira ${ }^{b}$ and Lucas Pizzuti ${ }^{\circledR}, a$
}

${ }^{a}$ Grupo de Pesquisa em Síntese e Caracterização Molecular do Mato Grosso do Sul, Universidade Federal da Grande Dourados, Rod. Dourados-Itahum, km 12, 79804-970 Dourados-MS, Brazil

${ }^{b}$ Faculdade de Ciências Biológicas e Ambientais, Universidade Federal da Grande Dourados, Rod. Dourados-Itahum, km 12, 79804-970 Dourados-MS, Brazil

'Laboratório de Materiais Inorgânicos, Departamento de Química, Universidade Federal de Santa Maria, Av. Roraima, 1000, 97105-900 Santa Maria-RS, Brazil

${ }^{d}$ Laboratório de Biotecnologia de Produtos Naturais e Sintéticos, Universidade de Caxias do Sul, Rua Francisco Getúlio Vargas, 1130, 95070-560 Caxias do Sul-RS, Brazil

eInstituto de Química e Geociências, Universidade Federal de Pelotas, Campus Universitário, 96010-900 Pelotas-RS, Brazil

\begin{abstract}
This work aims the synthesis of amidino pyrazolines by the cyclocondensation reaction between 3-(hetero)aryl-1-(2-hydroxyphenyl)enones and aminoguanidine hydrochloride under sonochemical conditions. Thirteen new compounds were synthesized in $15-83 \%$ of yield after sonication for 30 min without purification by chromatography. Furthermore, antimicrobial activities of the synthesized compounds were investigated by in vitro minimum inhibitory concentration (MIC) in a panel of four bacterial strains and four Candida yeasts. As result, the 5-(4-bromophenyl)3-(2-hydroxyphenyl)-4,5-dihydro-1H-pyrazole-1-carboximidamide hydrochloride was identified as the most active against gram-positive $S$. aureus and E. faecalis with MIC of $7.8 \mu \mathrm{g} \mathrm{mL}^{-1}$, similar to the value showed by chloramphenicol, which was used as standard.
\end{abstract}

Keywords: amidino pyrazoline, antibacterial activity, antifungal activity, cyclocondensation reaction, ultrasonic irradiation

\section{Introduction}

Pyrazoles and their non-aromatic analogous pyrazolines are important scaffolds for the development of new biologically active compounds. ${ }^{1}$ Although pyrazolecontaining natural products are rare, many synthetic molecules bearing this heterocycle are clinically approved pharmaceuticals such as celecoxib (anti-inflammatory activity), ${ }^{2}$ sildenafil (treatment of erectile dysfunction), ${ }^{3}$ and rimonabant (treatment of obesity), ${ }^{4}$ as well as agrochemicals such as chlorantraniliprole (insecticide), ${ }^{5}$

*e-mail: lucas.pizzuti@gmail.com fipronil (insecticide), ${ }^{6}$ and pyraclostrobin (fungicide). ${ }^{7}$ The related pyrazolines are motifs commonly found in compounds with potent bioactivities such as antibacterial, ${ }^{8}$ antifungal, ${ }^{9}$ antiviral, ${ }^{10}$ anti-inflamatory, ${ }^{11}$ antioxidant, ${ }^{12}$ anticancer ${ }^{13}$ antidepressant and antianxiety. ${ }^{14}$

In the same context, amidine-containing compounds show recognized bioactivities. Pentamidine is included on the World Health Organization's List of Essential Medicines for the basic health system indicated for the treatment of first stage human African tripanosomiasis and as a second-line therapy for leishmaniasis. ${ }^{15}$ In this concern, amidine-containing molecules have been pointed as prominent prototypes in the search for new antimicrobial agents. ${ }^{16}$ 
In the last years, the ultrasonic irradiation as green energy source has been applied in the field of pharmaceutical and green chemistry. The beneficial effect of sonication on organic synthesis are attributed to cavitation which creates special local conditions in the reaction medium. As result, reactions have been completed in shorter reaction times and products have been obtained in higher yields than under conventional methods. ${ }^{17}$ Recently, we demonstrated that ultrasonic irradiation was valuable in promoting the cyclocondensation reaction of chalcones and aminoguanidine hydrochloride for the synthesis of amidino pyrazolines. ${ }^{18}$ This method prompted us to synthesize series of pyrazolines in short reaction times and good yields using ethanol as green solvent despite the low solubility of aminoguanidine in such medium.

In continuation to this program, in this work we sought to promote the cyclocondensation reaction of phenolic chalcones and aminoguanidine hydrochloride under ultrasonic irradiation, aiming the synthesis of water soluble phenolic amidino pyrazolines hydrochlorides. Furthermore, searching for antimicrobial compounds, all new products were tested against gram-positive and gram-negative bacteria and Candida type yeasts.

\section{Experimental}

\section{General}

All the chemicals were used without purification as purchased from commercial suppliers (Sigma-Aldrich, St. Louis, USA). The sonicated reactions were carried out with a microtip probe connected to a $500 \mathrm{~W}$ Sonics Vibracell ultrasonic processor operating at $20 \mathrm{kHz}$ at $20 \%$ of the maximum power output. Melting point values were determined in open capillary on an Instrutherm DF-3600 II apparatus and are uncorrected. Infrared spectra (FTIR) were acquired on a JASCO-4100 spectrophotometer. ${ }^{1} \mathrm{H}$ and ${ }^{13} \mathrm{C}$ nuclear magnetic resonance (NMR) spectra were acquired on a Bruker Avance III HD instrument $(300 \mathrm{MHz}$ for ${ }^{1} \mathrm{H}$ and $75 \mathrm{MHz}$ for ${ }^{13} \mathrm{C}$ ) in $5 \mathrm{~mm}$ sample tubes at $298 \mathrm{~K}$ in dimethyl sulfoxide (DMSO- $d_{6}$ ). A hybrid highresolution and high accuracy microTof (Q-TOF) (Bruker ${ }^{\circledR}$ Scientific) was used for detection, with electrospray ionization (ESI) source (MicrOTOF-QII Bruker ${ }^{\circledR}$ Scientific) in positive mode. The compounds $\mathbf{3 a - 3} \mathbf{3}$ were dissolved individually in a solution of $50 \%(\mathrm{v} / \mathrm{v})$ chromatographic grade acetonitrile (Tedia, Fairfield, OH, USA), 50\% (v/v) deionized water and $0.1 \%$ formic acid. The solutions were infused directly into the ESI source by means of a syringe pump (Harvard Apparatus) at a flow rate of $180 \mu \mathrm{L} \mathrm{min}{ }^{-1}$. The drying temperature was $200{ }^{\circ} \mathrm{C}$ and nitrogen was used for drying gas, in a $10 \mathrm{~L} \mathrm{~min}^{-1}$ flow. The ESI(+)-MS and tandem ESI(+)-MS/MS were acquired under the following conditions: capillary and cone voltages were set to $+3500 \mathrm{~V}$ and $+40 \mathrm{~V}$, respectively, with a desolvation temperature of $100{ }^{\circ} \mathrm{C}$. For ESI(+)-MS/MS, the energy for the collision induced dissociations (CID) was optimized for each component. The software Compass DataAnalysis version 4.3 (Bruker ${ }^{\circledR}$ Scientific) was used for spectrum analysis. The diagnostic ions were identified by the comparison of their ESI(+)-MS/MS dissociation patterns with compounds with theoretical mass for the respective elementary formula. The data were collected in the $m / z$ range of 70-2000 at the speed of two scans per second, providing the resolution of 50,000 (full width at half maximum, FWHM) at $m / z 200$. No important ions were observed below $\mathrm{m} / \mathrm{z} 100$ or above $\mathrm{m} / \mathrm{z}, 700$, therefore ESI(+)-MS data is shown in the $\mathrm{m} / z, 100-700$ range. Diffraction data were collected on a Bruker D8 Venture Photon 100 diffractometer equipped with an Incoatec I $\mu \mathrm{S}$ high brilliance Mo-K $\alpha \mathrm{X}$ ray tube with twodimensional Montel micro-focusing optics. The structure was solved by direct methods using SHELXS. ${ }^{19}$ Subsequent Fourier-difference map analyses yielded the positions of the non-hydrogen atoms. Refinements were carried out with the SHELXL package. ${ }^{19}$ All refinements were made by fullmatrix least-squares on $\mathrm{F} 2$ with anisotropic displacement parameters for all non-hydrogen atoms. Hydrogen atoms were included in the refinement in calculated positions but the atoms (of hydrogens) that are commenting performing special bond were located in the Fourier map. Drawings were done using ORTEP-31 for Windows ${ }^{20}$ crystal data and more details of the data collection and refinements of the compound 3a is presented in Table 1 .

\section{Chemistry}

Procedure for the synthesis of 4,5-dihydro- $1 \mathrm{H}$-pyrazole1-carboximidamides 3a-m

A mixture of 3-(hetero)aryl-1-(2-hydroxyphenyl) enone 1a-1m (1.0 mmol), aminoguanidine hydrochloride 2 $(0.22 \mathrm{~g}, 2.0 \mathrm{mmol})$ and potassium hydroxide $(0.11 \mathrm{~g}$, $2.0 \mathrm{mmol})$ in ethanol $(15 \mathrm{~mL})$ was sonicated for $30 \mathrm{~min}$. After the excess of aminoguanidine hydrochloride had been filtered off, the resulting solution was quenched with $10 \%$ hydrogen chloride $(15 \mathrm{~mL})$. The mixture was extracted with chloroform $(3 \times 30 \mathrm{~mL})$ and the combined organic layer was dried over $\mathrm{MgSO}_{4}$. The solvent was evaporated and the residue was purified by recrystallization from ethyl acetate-methanol (10:1) to afford the 4,5-dihydro$1 H$-pyrazole-1-carboximidamides $\mathbf{3 a - 3}$ as crystalline solids. For the extraction of $\mathbf{3 m}$, after removal of excess 
Table 1. Crystal data and structure refinement for $\mathbf{3 a}$

\begin{tabular}{|c|c|}
\hline Chemical formula & $\mathrm{C}_{18} \mathrm{H}_{23} \mathrm{ClN}_{4} \mathrm{O}_{2}$ \\
\hline Molecular weight $/\left(\mathrm{g} \mathrm{mol}^{-1}\right)$ & 362.85 \\
\hline Crystal system, space group & monoclinic, $P 2_{1} / c$ \\
\hline Temperature / K & $100(2)$ \\
\hline$a, b, c / \AA$ & $\begin{array}{c}11.4290(5), 15.2989(9) \\
10.3794(5)\end{array}$ \\
\hline$\beta /$ degree & $102.713(2)$ \\
\hline Volume / $\AA^{3}$ & $1770.36(16)$ \\
\hline$Z, \mathrm{D}_{\text {calc }} /\left(\mathrm{g} \mathrm{cm}^{-3}\right)$ & $4,1.361$ \\
\hline Radiation type & Mo K $\alpha$ \\
\hline$\mu / \mathrm{mm}^{-1}$ & 0.236 \\
\hline Crystal size / mm & $0.20 \times 0.19 \times 0.18$ \\
\hline $\mathrm{F}(000)$ & 768 \\
\hline$\theta$ range / degree & $2.26-29.19$ \\
\hline $\mathrm{T}_{\min }, \mathrm{T}_{\max }$ & $0.9688,0.9444$ \\
\hline Index ranges & $\begin{array}{c}-15 \leq h \leq 15,-18 \leq k \leq 20 \\
-14 \leq l \leq 13\end{array}$ \\
\hline Reflections collected / unique & $10281 / 4774[\mathrm{R}(\mathrm{int})=0.0761]$ \\
\hline Completeness to theta maximum $/ \%$ & 99.6 \\
\hline Refinement method & full-matrix least-squares on $F^{2}$ \\
\hline Data / restraints / parameters & 4774 / 0 / 226 \\
\hline Goodness-of-fit (GOF) on $F^{2}$ & 1.082 \\
\hline Final $\mathrm{R}$ indices $[\mathrm{I}>2 \sigma(I)]$ & $R_{1}=0.0602, w R_{2}=0.1455$ \\
\hline $\mathrm{R}$ indices (all data) & $R_{1}=0.1199, w R_{2}=0.2111$ \\
\hline$\Delta \rho_{\max }, \Delta \rho_{\min } /\left(\mathrm{e} \AA^{-3}\right)$ & 0.315 and -0.379 \\
\hline
\end{tabular}

a-c: unit cell parameters; $Z$ : formula unit per unit cell; $\mathrm{D}_{\text {calc }}$ : calculated density; $\mu$ : absorption coefficient; $F(000)$ : structure factor in the zerothorder case; R(int): internal $R$-value; $F^{2}$ : squared structure factor; $R_{1}$ : $R$-value; I $>2 \sigma(\mathrm{I})$ : final $R$ indices based on the reflections observed; $w R_{2}: R$-value for $F^{2}$.

of aminoguanidine, the filtrate was treated with a saturated solution of $\mathrm{NaCl}$ and extracted with chloroform $(3 \times 30 \mathrm{~mL})$. The combined organic layer was acidified with some drops of concentrated hydrogen chloride, dried over $\mathrm{MgSO}_{4}$ and the solvent was evaporated.

3-(2-Hydroxyphenyl)-5-phenyl-4,5-dihydro-1 H-pyrazole1-carboximidamide (3a)

Whitish solid; 77\% yield; mp 294-296 ${ }^{\circ} \mathrm{C}$; FTIR (attenuated total reflectance, ATR) $v / \mathrm{cm}^{-1} 3282,3167$, 3105, 1646, 1615, 1596, 1585, 1493; ${ }^{1} \mathrm{H}$ NMR $(300 \mathrm{MHz}$, DMSO- $\left.d_{6}\right) \delta 10.00(\mathrm{~s}, 1 \mathrm{H}), 8.00(\mathrm{bs}, 4 \mathrm{H}), 7.78-7.75(\mathrm{~m}$, $1 \mathrm{H}), 7.40-7.28(\mathrm{~m}, 4 \mathrm{H}), 7.23-7.21(\mathrm{~m}, 2 \mathrm{H}), 7.05-7.02(\mathrm{~m}$, $1 \mathrm{H}), 6.92-6.87(\mathrm{~m}, 1 \mathrm{H}), 5.91-5.85\left(\mathrm{~m}, 1 \mathrm{H}, \mathrm{H}_{\mathrm{X}}\right), 4.12(\mathrm{dd}$, $\left.1 \mathrm{H}, J 18.4,11.2 \mathrm{~Hz}, \mathrm{H}_{\mathrm{M}}\right), 3.45-3.38\left(\mathrm{~m}, 1 \mathrm{H}, \mathrm{H}_{\mathrm{A}}\right) ;{ }^{13} \mathrm{C} \mathrm{NMR}$ $\left(75 \mathrm{MHz}, \mathrm{DMSO}-d_{6}\right) \delta 158.1,156.9,153.0,139.9,132.7$, 129.7, 129.1, 128.2, 125.3, 119.5, 117.1, 115.8, 59.4, 46.0; high resolution mass spectrometry (HRMS, ESI-TOF MS) $\mathrm{m} / \mathrm{z}$, calcd. for $\left[\mathrm{C}_{16} \mathrm{H}_{17} \mathrm{~N}_{4} \mathrm{O}\right]^{+}:$281.1397, found: 281.1393 .
3-(2-Hydroxyphenyl)-5-(o-tolyl)-4,5-dihydro-1 H-pyrazole1-carboximidamide (3b)

Yellowish solid; 64\% yield; mp 282-284 ${ }^{\circ} \mathrm{C}$; FTIR (ATR) $\mathrm{v} / \mathrm{cm}^{-1}$ 3273, 3117, 3032, 2940, 1646, 1617, 1590, 1490; ${ }^{1} \mathrm{H}$ NMR (300 MHz, DMSO- $d_{6}$ ) $\delta 9.97$ (s, 1H), 8.06 (bs, 4H), 7.77-7.73 (m, 1H), 7.35-7.13 (m, 4H), 7.04-7.01 $(\mathrm{m}, 1 \mathrm{H}), 6.91-6.86(\mathrm{~m}, 1 \mathrm{H}), 6.77-6.74(\mathrm{~m}, 1 \mathrm{H}), 5.91(\mathrm{dd}$, $\left.1 \mathrm{H}, J 11.2,2.9 \mathrm{~Hz}, \mathrm{H}_{\mathrm{X}}\right), 4.17\left(\mathrm{dd}, 1 \mathrm{H}, J 18.1,11.1 \mathrm{~Hz}, \mathrm{H}_{\mathrm{M}}\right)$, $3.27\left(\mathrm{dd}, 1 \mathrm{H}, J 18.1,2.9 \mathrm{~Hz}, \mathrm{H}_{\mathrm{A}}\right), 2.41(\mathrm{~s}, 3 \mathrm{H}) ;{ }^{13} \mathrm{C} \mathrm{NMR}$ $\left(75 \mathrm{MHz}, \mathrm{DMSO}-d_{6}\right) \delta 158.1,156.8,153.0,137.4,135.0$, $132.7,131.3,129.8,127.8,126.2$, 122.4, 119.5, 117.1, 115.7, 57.8, 44.9, 19.2; HRMS (ESI-TOF MS) $\mathrm{m} / \mathrm{z}$, calcd. for $\left[\mathrm{C}_{17} \mathrm{H}_{19} \mathrm{~N}_{4} \mathrm{O}\right]^{+}$: 295.1553, found: 295.1546.

\section{3-(2-Hydroxyphenyl)-5-( $p$-tolyl)-4,5-dihydro-1 H-pyrazole- 1-carboximidamide $\mathbf{( 3 b )}$}

Yellowish solid; $83 \%$ yield; mp 273-274 ${ }^{\circ} \mathrm{C}$; FTIR (ATR) $v / \mathrm{cm}^{-1}$ 3272, 3166, 3065, 2938, 1645, 1616, 1588, 1493; ${ }^{1} \mathrm{H}$ NMR $\left(300 \mathrm{MHz}, \mathrm{DMSO}-d_{6}\right) \delta 9.96$ (s, 1H), 7.96 (bs, 4H), 7.77-7.74 (m, 1H), 7.37-7.32 (m, 1H), 7.20-7.17 $(\mathrm{m}, 2 \mathrm{H}), 7.12-7.09(\mathrm{~m}, 2 \mathrm{H}), 7.04-7.01(\mathrm{~m}, 1 \mathrm{H}), 6.93-6.88$ (m, 1H), 5.81 (dd, 1H, J 11.2, $3.0 \mathrm{~Hz}, \mathrm{H}_{\mathrm{X}}$ ), 4.09 (dd, 1H, $\left.J 18.3,11.1 \mathrm{~Hz}, \mathrm{H}_{\mathrm{M}}\right), 3.37-3.36\left(\mathrm{~m}, 1 \mathrm{H}, \mathrm{H}_{\mathrm{A}}\right), 2.26(\mathrm{~s}, 3 \mathrm{H})$; ${ }^{13} \mathrm{C}$ NMR (75 MHz, DMSO- $\left.d_{6}\right) \delta 158.0,156.8,152.9$, $137.5,136.9,132.6,129.7,129.6,125.2,119.5,117.1$, 115.8, 59.3, 45.9, 20.7; HRMS (ESI-TOF MS) m/z, calcd. for $\left[\mathrm{C}_{17} \mathrm{H}_{19} \mathrm{~N}_{4} \mathrm{O}\right]^{+}$: 295.1553, found: 295.1551.

3-(2-Hydroxyphenyl)-5-(2-methoxyphenyl)-4,5-dihydro$1 \mathrm{H}$-pyrazole-1-carboximidamide (3d)

Yellowish solid; $73 \%$ yield; mp 290-293 ${ }^{\circ} \mathrm{C}$; FTIR (ATR) v / cm $\mathrm{cm}^{-1} 3309,3194,3108,2966,2939,1644,1618$, 1594, 1494; ${ }^{1} \mathrm{H}$ NMR (300 MHz, DMSO- $d_{6}$ ) $\delta 10.00$ (s, $1 \mathrm{H}), 7.96$ (bs, 4H), 7.76-7.73 (m, 1H), 7.35-7.29 (m, 2H), 7.10-7.02 (m, 2H), 6.96-6.86 (m, 3H), $5.84(\mathrm{dd}, 1 \mathrm{H}$, $\left.J 11.1,3.1 \mathrm{~Hz}, \mathrm{H}_{\mathrm{X}}\right), 4.07$ (dd, $1 \mathrm{H}, J 18.3,11.2 \mathrm{~Hz}, \mathrm{H}_{\mathrm{M}}$ ), $3.43(\mathrm{~s}, 3 \mathrm{H}), 3.31\left(\mathrm{dd}, 1 \mathrm{H}, J 18.3,3.2 \mathrm{~Hz}, \mathrm{H}_{\mathrm{A}}\right) ;{ }^{13} \mathrm{C} \mathrm{NMR}$ $\left(75 \mathrm{MHz}, \mathrm{DMSO}-d_{6}\right) \delta 158.6,156.8,156.2,153.0,132.5$, 129.6, 129.5, 126.6, 125.0, 120.3, 119.4, 117.0, 115.8, 111.8, 56.3, 55.7, 44.7; HRMS (ESI-TOF MS) $\mathrm{m} / z$, calcd. for $\left[\mathrm{C}_{17} \mathrm{H}_{19} \mathrm{~N}_{4} \mathrm{O}_{2}\right]^{+}:$311.1503, found: 311.1494 .

3-(2-Hydroxyphenyl)-5-(4-methoxyphenyl)-4,5-dihydro$1 \mathrm{H}$-pyrazole-1-carboximidamide (3e)

Yellowish solid; $80 \%$ yield; mp 274-276 ${ }^{\circ} \mathrm{C}$; FTIR (ATR) $\mathrm{v} / \mathrm{cm}^{-1}$ 3266, 3178, 3066, 2953, 2835, 1645, 1615, 1587, 1510, 1492; ${ }^{1} \mathrm{H}$ NMR (300 MHz, DMSO- $\left.d_{6}\right) \delta 9.98$ (s, 1H), $7.96(\mathrm{bs}, 4 \mathrm{H}), 7.78-7.74(\mathrm{~m}, 1 \mathrm{H}), 7.38-7.32(\mathrm{~m}$, 2H), 7.18-7.15 (m, 2H), 7.04-7.02 (m, 1H), 6.95-6.89 (m, $3 \mathrm{H}), 5.82-5.77\left(\mathrm{~m}, 1 \mathrm{H}, \mathrm{H}_{\mathrm{X}}\right), 4.08(\mathrm{dd}, 1 \mathrm{H}, J 18.3,11.1 \mathrm{~Hz}$, $\left.\mathrm{H}_{\mathrm{M}}\right), 3.72(\mathrm{~s}, 3 \mathrm{H}), 3.38-3.37\left(\mathrm{~m}, 1 \mathrm{H}, \mathrm{H}_{\mathrm{A}}\right) ;{ }^{13} \mathrm{C} \mathrm{NMR}$ 
(75 MHz, DMSO- $\left.d_{6}\right) \delta 159.1,158.1,156.8,152.9,132.6$, 131.8, 129.7, 126.7, 119.5, 117.1, 115.9, 114.4, 59.0, 55.2, 45.9; HRMS (ESI-TOF MS) $m / z$, calcd. for $\left[\mathrm{C}_{17} \mathrm{H}_{19} \mathrm{~N}_{4} \mathrm{O}_{2}\right]^{+}$: 311.1503, found: 311.1485 .

5-(4-Fluorophenyl)-3-(2-hydroxyphenyl)-4,5-dihydro$1 \mathrm{H}$-pyrazole-1-carboximidamide (3f)

Whitish solid; 74\% yield; mp 272-275 ${ }^{\circ} \mathrm{C}$; FTIR (ATR) $\mathrm{v} / \mathrm{cm}^{-1}$ 3363, 3275, 3080, 2949, 2829, 1647, 1615, 1594, 1509, 1492; ${ }^{1} \mathrm{H}$ NMR (300 MHz, DMSO- $\left.d_{6}\right) \delta 9.98(\mathrm{~s}$, $1 \mathrm{H}), 8.00$ (bs, 4H), 7.78-7.75 (m, 1H), 7.38-7.19 (m, 5H), 7.04-7.01 (m, 1H), 6.93-6.88 (m, 1H), 5.87 (dd, 1H, J 11.3, $\left.3.0 \mathrm{~Hz}, \mathrm{H}_{\mathrm{X}}\right), 4.12\left(\mathrm{dd}, 1 \mathrm{H}, J 18.5,11.2 \mathrm{~Hz}, \mathrm{H}_{\mathrm{M}}\right), 3.39-3.38$ $\left(\mathrm{m}, 1 \mathrm{H}, \mathrm{H}_{\mathrm{A}}\right) ;{ }^{13} \mathrm{C}$ NMR $\left(75 \mathrm{MHz}, \mathrm{DMSO}-d_{6}\right) \delta 161.8(\mathrm{~d}$, $J 244.4 \mathrm{~Hz}), 158.0,156.8,152.9,136.1$ (d, J 3.0 Hz), 132.7, 129.7, 127.6 (d, J 8.4 Hz), 119.5, 117.1, 115.9 (d, $J 21.7 \mathrm{~Hz}$ ), 115.8, 58.8, 44.9; HRMS (ESI-TOF MS) $m / z$, calcd. for $\left[\mathrm{C}_{16} \mathrm{H}_{16} \mathrm{FN}_{4} \mathrm{O}\right]^{+}$: 299.1303, found: 299.1301 .

5-(2-Bromophenyl)-3-(2-hydroxyphenyl)-4,5-dihydro$1 \mathrm{H}$-pyrazole-1-carboximidamide $\mathbf{( 3 g})$

Whitish solid; 60\% yield; mp 279-282 ${ }^{\circ} \mathrm{C}$; FTIR (ATR) $\mathrm{v} / \mathrm{cm}^{-1}$ 3352, 3274, 3196, 3141, 3064, 2957, 2838, 1656, $1613,1592,1494 ;{ }^{1} \mathrm{H}$ NMR $\left(300 \mathrm{MHz}, \mathrm{DMSO}-d_{6}\right) \delta 10.10$ (s, 1H), 8.07 (bs, 4H), 7.83-7.72 (m, 2H), 7.44-7.39 (m, $1 \mathrm{H}), 7.35-7.26(\mathrm{~m}, 2 \mathrm{H}), 7.03-7.00(\mathrm{~m}, 1 \mathrm{H}), 6.93-6.85$ $(\mathrm{m}, 2 \mathrm{H}), 5.84\left(\mathrm{dd}, 1 \mathrm{H}, J 11.2,3.0 \mathrm{~Hz}, \mathrm{H}_{\mathrm{X}}\right), 4.24(\mathrm{dd}, 1 \mathrm{H}$, $J$ 18.4, $\left.11.1 \mathrm{~Hz}, \mathrm{H}_{\mathrm{M}}\right), 3.40-3.34\left(\mathrm{~m}, 1 \mathrm{H}, \mathrm{H}_{\mathrm{A}}\right) ;{ }^{13} \mathrm{C} \mathrm{NMR}$ $\left(75 \mathrm{MHz}, \mathrm{DMSO}-d_{6}\right) \delta 157.7,156.9,153.1,137.9,133.8$, $132.7,130.0,129.6,128.2,125.1,121.5,119.4,117.1$, 115.7, 60.1, 45.5; HRMS (ESI-TOF MS) $\mathrm{m} / z$, calcd. for $\left[\mathrm{C}_{16} \mathrm{H}_{16} \mathrm{BrN}_{4} \mathrm{O}\right]^{+}:$359.0502, found: 359.0498 .

5-(3-Bromophenyl)-3-(2-hydroxyphenyl)-4,5-dihydro$1 \mathrm{H}$-pyrazole-1-carboximidamide (3h)

Yellowish solid; 44\% yield; mp 308-309 ${ }^{\circ} \mathrm{C}$; FTIR (ATR) $v / \mathrm{cm}^{-1} 3313,3178,3121,2934,2847,1638,1615$, 1603, 1590, 1494; ${ }^{1} \mathrm{H}$ NMR (300 MHz, DMSO- $\left.d_{6}\right) \delta 10.00$ $(\mathrm{s}, 1 \mathrm{H}), 8.03(\mathrm{bs}, 4 \mathrm{H}), 7.78-7.75(\mathrm{~m}, 1 \mathrm{H}), 7.55-7.51(\mathrm{~m}$, $1 \mathrm{H}), 7.46-7.45(\mathrm{~m}, 1 \mathrm{H}), 7.39-7.32(\mathrm{~m}, 2 \mathrm{H}), 7.19-7.17(\mathrm{~m}$, $1 \mathrm{H})$, 7.04-7.01 (m, 1H), 6.93-6.88 (m, 1H), $5.86(\mathrm{dd}, 1 \mathrm{H}$, $\left.J 11.0,3.0 \mathrm{~Hz}, \mathrm{H}_{\mathrm{X}}\right), 4.13\left(\mathrm{dd}, 1 \mathrm{H}, J 18.5,11.3 \mathrm{~Hz}, \mathrm{H}_{\mathrm{M}}\right.$ ), $3.31\left(\mathrm{~m}, 1 \mathrm{H}, \mathrm{H}_{\mathrm{A}}\right) ;{ }^{13} \mathrm{C} \mathrm{NMR}\left(75 \mathrm{MHz}, \mathrm{DMSO}-d_{6}\right) \delta 158.0$, $156.8,152.9,142.4,132.7,131.4,131.1,129.7,128.5$, $124.1,122.0,119.5,117.1,115.7,58.9,45.9$; HRMS (ESI-TOF MS) $m / z$, calcd. for $\left[\mathrm{C}_{16} \mathrm{H}_{16} \mathrm{BrN}_{4} \mathrm{O}\right]^{+}: 359.0502$, found: 359.0501 .

5-(4-Bromophenyl)-3-(2-hydroxyphenyl)-4,5-dihydro$1 \mathrm{H}$-pyrazole-1-carboximidamide (3i)

Yellowish solid; $68 \%$ yield; mp 277-279 ${ }^{\circ} \mathrm{C}$; FTIR
(ATR) $\mathrm{v} / \mathrm{cm}^{-1} 3394,3192,3101,2956,2836,1656,1617$, 1590, 1490; ${ }^{1} \mathrm{H}$ NMR $\left(300 \mathrm{MHz}, \mathrm{DMSO}-d_{6}\right) \delta 9.99(\mathrm{~s}, 1 \mathrm{H})$, 8.01 (bs, 4H), 7.78-7.75 (m, 1H), 7.61-7.58 (m, 2H), 7.37$7.32(\mathrm{~m}, 1 \mathrm{H}), 7.19-7.15(\mathrm{~m}, 2 \mathrm{H}), 7.03-7.01(\mathrm{~m}, 1 \mathrm{H}), 6.93-$ $6.88(\mathrm{~m}, 1 \mathrm{H}), 5.85\left(\mathrm{dd}, 1 \mathrm{H}, J 11.2,3.0 \mathrm{~Hz}, \mathrm{H}_{\mathrm{X}}\right), 4.13(\mathrm{dd}$, $\left.1 \mathrm{H}, J 18.4,11.3 \mathrm{~Hz}, \mathrm{H}_{\mathrm{M}}\right), 3.46-3.38\left(\mathrm{~m}, 1 \mathrm{H}, \mathrm{H}_{\mathrm{A}}\right) ;{ }^{13} \mathrm{C} \mathrm{NMR}$ $\left(75 \mathrm{MHz}, \mathrm{DMSO}-d_{6}\right) \delta 158.0,156.9,152.9,139.2,132.7$, 132.0, 129.7, 121.3, 119.5, 117.1, 115.8, 58.9, 45.8; HRMS (ESI-TOF MS) $m / z$, calcd. for $\left[\mathrm{C}_{16} \mathrm{H}_{16} \mathrm{BrN}_{4} \mathrm{O}\right]^{+}: 359.0502$, found: 359.0492 .

3-(2-Hydroxyphenyl)-5-(4-(trifluoromethyl)phenyl)4,5-dihydro- $1 \mathrm{H}$-pyrazole-1-carboximidamide (3j)

Whitish solid; $63 \%$ yield; mp 294-296 ${ }^{\circ} \mathrm{C}$; FTIR (ATR) $v / \mathrm{cm}^{-1} 3393,3289,3222,3153,3079,2943,2843,1657$, 1616, 1592, 1495; ${ }^{1} \mathrm{H}$ NMR (300 MHz, DMSO- $\left.d_{6}\right) \delta 9.93$ (bs, 1H), 8.04 (bs, 4H), 7.79-7.77 (m, 3H), 7.45-7.43 (m, $2 \mathrm{H}), 7.38-7.32(\mathrm{~m}, 1 \mathrm{H}), 7.03-7.01(\mathrm{~m}, 1 \mathrm{H}), 6.93-6.88(\mathrm{~m}$, $1 \mathrm{H}), 5.98$ (dd, $1 \mathrm{H}, J 11.4,3.0 \mathrm{~Hz}, \mathrm{H}_{\mathrm{X}}$ ), 4.18 (dd, $1 \mathrm{H}, J 18.5$, $\left.11.4 \mathrm{~Hz}, \mathrm{H}_{\mathrm{M}}\right), 3.49-3.40\left(\mathrm{~m}, 1 \mathrm{H}, \mathrm{H}_{\mathrm{A}}\right) ;{ }^{13} \mathrm{C} \mathrm{NMR}(75 \mathrm{MHz}$, DMSO- $\left.d_{6}\right) \delta 157.9,156.9,153.0,144.3,132.7,129.7$, 128.7 (q, $J 31.7 \mathrm{~Hz}$ ), 126.3, 126.0 (q, J 4.0 Hz), 124.1 (q, J 272.4 Hz), 119.4, 117.1, 115.7, 59.1, 45.9; HRMS (ESI-TOF MS) $m / z$, calcd. for $\left[\mathrm{C}_{17} \mathrm{H}_{16} \mathrm{~F}_{3 \mathrm{~N} 4} \mathrm{O}\right]^{+}:$349.1271, found: 349.1252 .

3-(2-Hydroxyphenyl)-5-(3-nitrophenyl)-4,5-dihydro1 H-pyrazole-1-carboximidamide (3k)

Brownish solid; $15 \%$ yield; mp $311-312{ }^{\circ} \mathrm{C}$; FTIR (ATR) $\mathrm{v} / \mathrm{cm}^{-1}$ 3296, 3208, 3103, 2934, 2864, 1645, 1616, 1603, 1529, 1494; ${ }^{1} \mathrm{H}$ NMR (300 MHz, DMSO-d $\left.d_{6}\right) \delta 10.02$ (s, 1H), 8.21-8.18 (m, 1H), 8.11-8.10 (m, 1H), 8.06 (bs, $4 \mathrm{H}), 7.80-7.64(\mathrm{~m}, 3 \mathrm{H}), 7.38-7.32(\mathrm{~m}, 1 \mathrm{H}), 7.03-7.00(\mathrm{~m}$, $1 \mathrm{H}), 6.94-6.88(\mathrm{~m}, 1 \mathrm{H}), 6.02\left(\mathrm{dd}, 1 \mathrm{H}, J 11.4,3.2 \mathrm{~Hz}, \mathrm{H}_{\mathrm{X}}\right)$, 4.20 (dd, 1H, J 18.6, 11.4 Hz, $\mathrm{H}_{\mathrm{M}}$ ), 3.53-3.46 (m, 1H, $\mathrm{H}_{\mathrm{A}}$ ); ${ }^{13} \mathrm{C}$ NMR (75 MHz, DMSO- $\left.d_{6}\right) \delta 158.0,156.9,152.9$, $148.0,141.8,132.8,132.0,130.9,129.7,123.2,120.7$, 119.5, 117.1, 115.7, 58.9, 46.0; HRMS (ESI-TOF MS) $m / z$, calcd. for $\left[\mathrm{C}_{16} \mathrm{H}_{16} \mathrm{~N}_{5} \mathrm{O}_{3}\right]^{+}: 326.1248$, found: 326.1245 .

3-(2-Hydroxyphenyl)-5-(thiophen-2-yl)-4,5-dihydro$1 \mathrm{H}$-pyrazole-1-carboximidamide (3I)

Yellowish solid; $78 \%$ yield; mp $264-267{ }^{\circ} \mathrm{C}$; FTIR (ATR) v / cm ${ }^{-1} 3321,3200,3107,2940,2869,1615,1589$, 1493; ${ }^{1} \mathrm{H}$ NMR $\left(300 \mathrm{MHz}, \mathrm{DMSO}-d_{6}\right) \delta 10.00(\mathrm{~s}, 1 \mathrm{H}), 8.04$ (bs, 4H), 7.81-7.78 (m, 1H), 7.49-7.47 (m, 1H), 7.39-7.34 (m, 1H), 7.22-7.21 (m, 1H), 7.04-6.90 (m, 3H), 6.22 (dd, 1H, J 10.8, $2.5 \mathrm{~Hz}, \mathrm{H}_{\mathrm{X}}$ ), 4.08 (dd, 1H, J 18.4, $10.8 \mathrm{~Hz}$, $\left.\mathrm{H}_{\mathrm{M}}\right), 3.60\left(\mathrm{dd}, 1 \mathrm{H}, J 18.3,2.5 \mathrm{~Hz}, \mathrm{H}_{\mathrm{A}}\right) ;{ }^{13} \mathrm{C} \mathrm{NMR}(75 \mathrm{MHz}$, DMSO- $\left.d_{6}\right) \delta 158.2,156.9,152.9,141.9,132.8,129.7$, 126.9, 126.5, 126.2, 119.5, 117.1, 115.8, 55.9, 45.8; HRMS 
(ESI-TOF MS) $m / z$, calcd. for $\left[\mathrm{C}_{14} \mathrm{H}_{15} \mathrm{~N}_{4} \mathrm{OS}\right]^{+}: 287.0961$, found: 287.0954 .

3-(2-Hydroxyphenyl)-5-(pyridin-2-yl)-4,5-dihydro$1 \mathrm{H}$-pyrazole-1-carboximidamide $(3 \mathrm{~m})$

Whitish solid; $24 \%$ yield; $m p 276-278^{\circ} \mathrm{C}$; FTIR (ATR) $v / \mathrm{cm}^{-1} 3332,3262,3208,3097,3051,2813,2720,1670$, $1618,1589,1495 ;{ }^{1} \mathrm{H}$ NMR $\left(300 \mathrm{MHz}, \mathrm{DMSO}-d_{6}\right) \delta 9.97$ (s, 1H), $8.05(\mathrm{bs}, 4 \mathrm{H}), 8.03-8.00(\mathrm{~m}, 2 \mathrm{H}), 7.77-7.74(\mathrm{~m}$, $1 \mathrm{H}), 7.57-7.50(\mathrm{~m}, 2 \mathrm{H}), 7.37-7.31(\mathrm{~m}, 1 \mathrm{H}), 7.04-7.01$ $(\mathrm{m}, 1 \mathrm{H}), 6.92-6.87(\mathrm{~m}, 1 \mathrm{H}), 6.02(\mathrm{dd}, 1 \mathrm{H}, J 11.5,3.3 \mathrm{~Hz}$, $\left.\mathrm{H}_{\mathrm{X}}\right), 4.15\left(\mathrm{dd}, 1 \mathrm{H}, J 18.4,11.5 \mathrm{~Hz}, \mathrm{H}_{\mathrm{M}}\right), 3.61(\mathrm{dd}, 1 \mathrm{H}$, $J$ 18.4, $\left.3.3 \mathrm{~Hz}, \mathrm{H}_{\mathrm{A}}\right) ;{ }^{13} \mathrm{C}$ NMR $\left(75 \mathrm{MHz}\right.$, DMSO- $\left.d_{6}\right) \delta$ 157.7, 156.8, 156.3, 153.3, 148.2, 139.6, 132.6, 129.7, 124.2, 121.9, 119.5, 117.1, 115.7, 59.5, 44.5; HRMS (ESI-TOF MS) $\mathrm{m} / \mathrm{z}$, calcd. for $\left[\mathrm{C}_{15} \mathrm{H}_{10} \mathrm{~N}_{5} \mathrm{O}\right]^{+}: 282.1349$, found: 282.1330 .

\section{Biology}

The minimum inhibitory concentrations (MIC) of the 4,5-dihydro- $1 H$-pyrazole-1-carboximidamides 3a-3m were determined by the broth microdilution test, following the guidelines of the Clinical and Laboratory Standards Institute (2012) and document M27-A3 with some adaptations. ${ }^{21}$ The microorganisms are strains from American Type Culture Collection. Two gram-negative bacteria: Salmonella enteritidis (ATCC 13076) and Escherichia coli (ATCC 25922); and two gram-positive: Staphylococcus aureus (ATCC 25923) and Enterococcus faecalis (ATCC 51299), in addition to four yeasts: Candida krusei (ATCC 6558), Candida tropicalis (ATCC 750), Candida albicans (ATCC 90028) and Candida glabrata (ATCC 2001) were used. Dimethyl sulfoxide was used as solvent for the antimicrobial tests.

\section{Results and Discussion}

\section{Synthesis and structural characterization}

3-(Hetero)aryl-1-(2-hydroxyphenyl)enones 1a-1m were prepared according to previously reported procedures. ${ }^{22}$ Sonication of $1 \mathbf{a}-1 \mathbf{m}$ and aminoguanidine hydrochloride 2 for $30 \mathrm{~min}$ in the presence of potassium hydroxide using ethanol as solvent, and further treatment with $10 \%$ hydrogen chloride before extraction, afforded the novel 4,5-dihydro$1 H$-pyrazole-1-carboximidamides hydrochlorides $\mathbf{3 a - 3 m}$ in acceptable to good yields (15-83\%) (Scheme 1). The method determined here showed some advantages including the short reaction time, non-chromatographic purification and hydroxyl protection/deprotection steps not required. Unfortunately, all the new compounds were water-insoluble. Consequently, dimethyl sulfoxide was used as the solvent for the bactericidal and fungicidal tests.

The mechanism of the cyclocondensation reaction between aminoguanidine and $\alpha, \beta$-unsaturated ketones for synthesis of amidino pyrazolines remains experimentally unestablished. However, we propose the mechanism showed in Scheme 2 based on a previously reported mechanism ${ }^{23}$ that considered the hard and soft acids and bases (HSAB) concept and the structure of aminoguanidine obtained by quantum chemical studies. ${ }^{24}$ In the first step, the $\mathrm{KOH}$ neutralizes the aminoguanidine salt $\mathbf{2}$ to give aminoguanidine free base (tautomer forms). In the same line, the phenol is deprotonated by the $\mathrm{KOH}$ leading to the formation of the phenoxide intermediate $\mathbf{I}$. In the next step, the Aza-Michael-type addition with the internal imine nitrogen gives the intermediate II. Subsequently, III is formed after an intramolecular nucleophilic attack by nitrogen, which is dehydrated to give the product IV. The product $\mathbf{3}$ is formed after the reaction media is acidified.

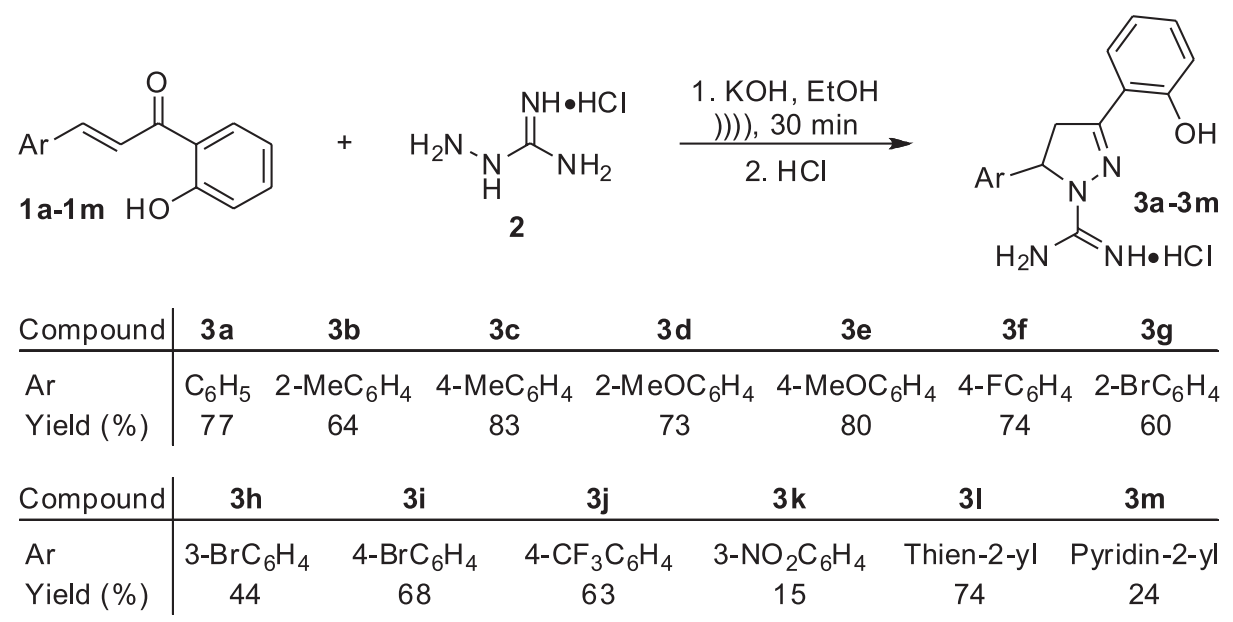

Scheme 1. Ultrasound-promoted synthesis of 4,5-dihydro- $1 H$-pyrazole-1-carboximidamides $\mathbf{3 a - 3 m}$. 


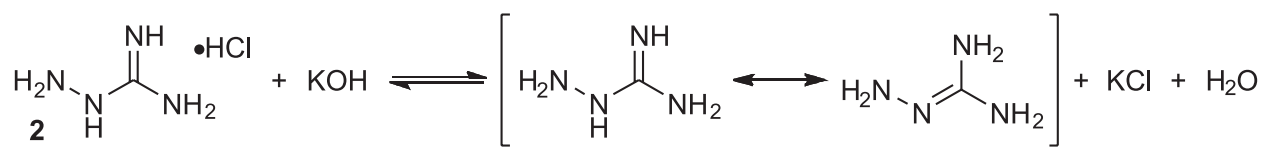<smiles>CC=CC(=O)C1=CC=[C+]C=C1O</smiles><smiles>NC(N)=N/C=C/C(=O)C1=CCCC=C1O</smiles><smiles>C=CC</smiles>

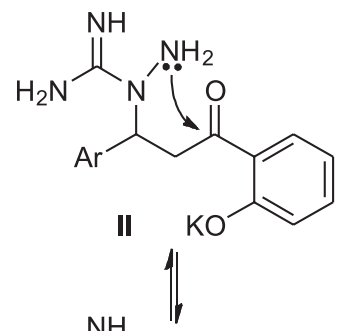<smiles>N/C(=N/Cl)N1N=C(c2ccccc2O)CC1[Hg]Cl</smiles><smiles>N=C(N)N1N=C(c2ccccc2O)CC1[14CH2]O</smiles><smiles>N=C(N)N1NC(O)(c2ccccc2O)CC1[125I]</smiles>

Scheme 2. Proposed mechanism for the cyclocondensation reaction between 3-(hetero)aryl-1-(2-hydroxyphenyl)enones and aminoguanidine hydrochloride in the presence of $\mathrm{KOH}$.

The structure elucidation of compounds $\mathbf{3 a - 3} \mathbf{m}$ was performed from analysis of their spectroscopic and spectrometric data (FTIR, ${ }^{1} \mathrm{H}$ NMR, ${ }^{13} \mathrm{C}$ NMR and HRMS). The IR spectrum of pyrazole $\mathbf{3 d}$ taken as representative example of the series showed a pair of absorption bands at 3309 and $3194 \mathrm{~cm}^{-1}$ related to the $\mathrm{N}-\mathrm{H}$ of protonated amidine group. No prominent band was seen in the region where stretching vibration of the $\mathrm{OH}$ oscillator should appear. The ${ }^{1} \mathrm{H}$ NMR spectrum of $\mathbf{3 d}$ showed a singlet at $10.00 \mathrm{ppm}$ assigned to the $\mathrm{OH}$ proton and a broad singlet at $7.96 \mathrm{ppm}$ associated to the protons of the protonated amidine group. The signals attributed to the eight aromatic protons appear in the region 7.76-6.86 ppm. The signals assigned to the protons of the AMX system appear as three double-doublets at $3.31 \mathrm{ppm}$ with coupling constant values ${ }^{2} J=18.3 \mathrm{~Hz}$ and ${ }^{3} J=3.2 \mathrm{~Hz}$ for proton $\mathrm{H}_{\mathrm{A}} ; 4.07 \mathrm{ppm}$, with coupling constant values ${ }^{2} J=18.3 \mathrm{~Hz}$ and ${ }^{3} J=11.2 \mathrm{~Hz}$, for proton $\mathrm{H}_{\mathrm{M}}$; and $5.84 \mathrm{ppm}$, with coupling constant values ${ }^{3} J=11.1 \mathrm{~Hz}$ and ${ }^{3} J=3.1 \mathrm{~Hz}$, for proton $\mathrm{H}_{\mathrm{X}}$. The methoxy group generated a singlet at $3.43 \mathrm{ppm}$. Furthermore, the ${ }^{13} \mathrm{C}$ NMR spectrum showed all the expected signals.

The thirteen products $\mathbf{3 a - 3 m}$ were analyzed by HRMS with electrospray ionization (ESI). Spectra were acquired in positive mode ESI(+)-MS and in ESI(+)-MS/MS for evaluation of fragmentation pathway. The exact mass/ charge $(\mathrm{m} / \mathrm{z})$ of the hydrogen adduct $[\mathrm{M}+\mathrm{H}]^{+}$of all compounds are present with errors less than $10 \mathrm{ppm}$ relative to that theoretically expected for elemental formula, as for instance for the $\mathbf{3 d}$ with $\mathrm{m} / \mathrm{z} 311.1494$ for the formula $\left[\mathrm{C}_{17} \mathrm{H}_{18} \mathrm{~N}_{4} \mathrm{O}_{2}+\mathrm{H}\right]^{+}$(theoretical $\mathrm{m} / z 311.1503$ ). Furthermore, for some compounds it is possible to identify the dimers formation, as for instance, with the $m / z 621.2867$ and 657.2620, which are the $[\mathrm{M}+\mathrm{M}+\mathrm{H}]^{+}$and $\left[\mathrm{M}+\mathrm{M}+2 \mathrm{H}_{2} \mathrm{O}\right]^{+}$, respectively, for the compound $\mathbf{3 d}$. In the same line, the fit of isotopic ratio (theoretical versus experimental) assist in the confirmation of elementary composition of synthesized compounds, being in the $\mathbf{3 d}$ example equal to $23.6 \mathrm{msig}$. Errors of exact $\mathrm{m} / \mathrm{z}$ below $10 \mathrm{ppm}$, as well as differences of isotopic ratios less than $25 \mathrm{msig}$, for HRMS with ionization by ESI and mass separation by quadrupole-time of flight (Q-TOF), confirm the elemental composition. ${ }^{25}$ Moreover, the fragmentation pathway has important information about the molecular structure. For the title compounds, it is similar to that proposed by Kéki et al., ${ }^{26}$ for 1,3,5-trisubstituted pyrazolines, as well as dos Santos et al. ${ }^{27}$ for dihydropyrimidines. The Figure 1 demonstrates this HRMS tools used to characterize the synthesized amidino pyrazolines, using as example the compound 3d. For the other compounds, see the Supplementary Information (SI) section.

Finally, the molecular structure of compound 3a was confirmed by single X-ray diffraction analysis (Figure 2). It shows a protonated amidino-nitrogen atom resulting in a delocalized positive charge in the N3-C4-N4 system, according to the symmetric bond length between $\mathrm{N} 3-\mathrm{C} 4=1.325(4) \AA$ and $\mathrm{C} 4-\mathrm{N} 4=1.323(4) \AA$. These values 


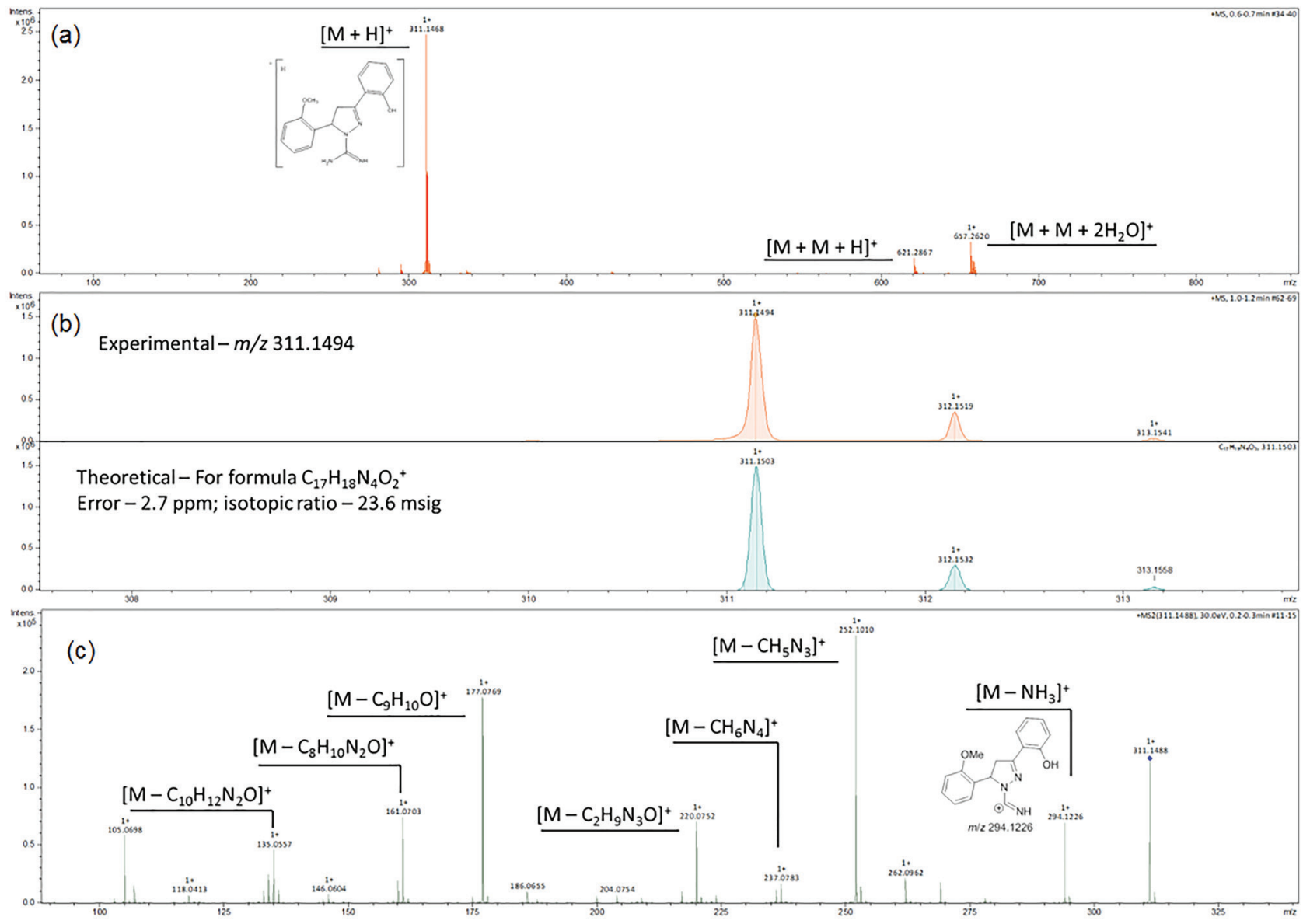

Figure 1. The HRMS analysis of compound 3d. In (a) the full spectra; in (b) the expansion between 308 and 314 u.a.m. highlighting the exact mass and isotopic ratio; and in (c) the analysis in MS-MS mode (fragmentation pathway).

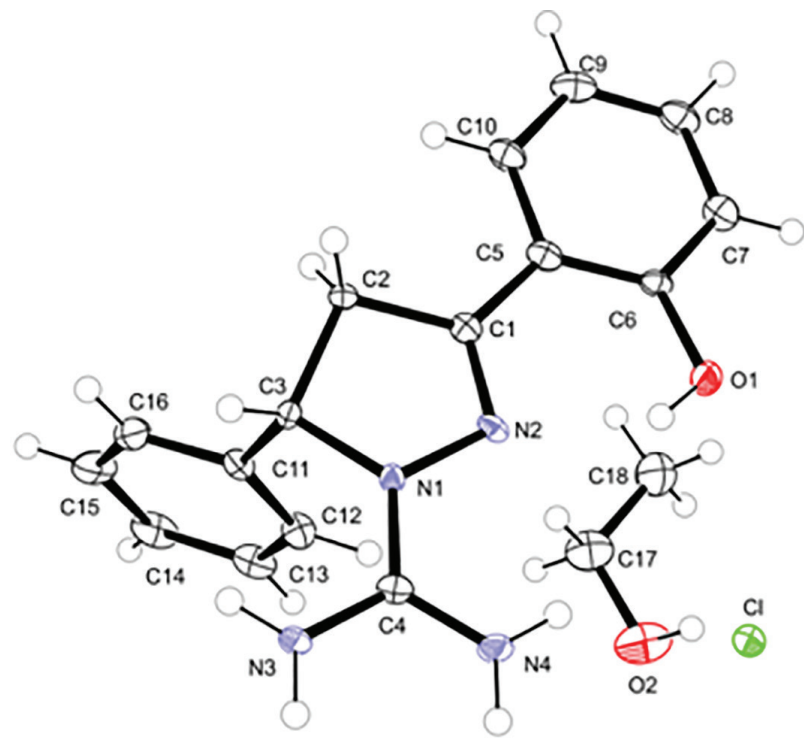

Figure 2. Molecular structure of 3a. Thermal ellipsoids drawn at the $50 \%$ probability level.

are intermediate between single and double $\mathrm{C}-\mathrm{N}$ bond lengths and are very close to those reported for the protonated amidino group in the aminoguanidine hydrochloride structure.$^{28}$ The electroneutrality is achieved by a chloride anion. Additionally, an ethanol molecule is observed as a solvate. Crystal data, data collection and structure refinement details are summarized in Table 1. Other crystallographic data for 3a are shown in the SI section.

The one-dimensional (1-D) growth of 3a in the $b c$ plane is represented in Figure 3. The intermolecular $\mathrm{H}$-bonds of type $\mathrm{N}-\mathrm{H}^{\cdots} \mathrm{Cl}^{-}$involving the chloride ion and the hydrogen of the protonated amidino group measure between 2.3801(7) and 2.7397(7) $\AA$. For the interactions of type $\mathrm{O}-\mathrm{H} \cdots \mathrm{Cl}^{-}$, the distances are 2.8394(7) $\AA$ for the one involving the phenolic hydrogen and 2.5001(8) $\AA$ for the ethanolic one. The intramolecular $\mathrm{H}$-bonds of type $\mathrm{O}-\mathrm{H} \cdots \mathrm{N}$ between pyrazoline nitrogen and phenolic hydrogen measure 2.0531(24). . These lengths are shorter than the sum of the $\mathrm{Cl} / \mathrm{H}, \mathrm{O} / \mathrm{H}$ and $\mathrm{N} / \mathrm{H}$ van der Waals radii (3.0, 2.67 and $2.75 \AA$, respectively). ${ }^{29}$

\section{Antibacterial activity}

All the novel compounds were screened for their in vitro antibacterial activity against gram-positive 


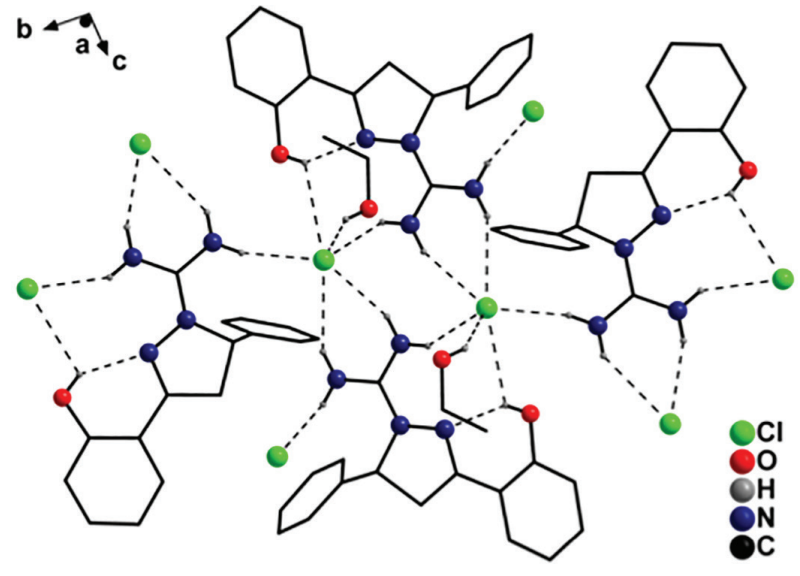

Figure 3. Supramolecular assembly of $\mathbf{3 a}$ in the $b c$ plane and the intermolecular $\mathrm{Cl} \cdots \mathrm{H}$ and $\mathrm{N} \cdots \mathrm{H}$ interactions (dotted lines). For clarity, some of the hydrogen atoms are not shown.

and gram-negative bacteria. The minimum inhibitory concentrations (MIC) were determined and reported in Table 2. Gram-negative bacteria E. coli and S. Enteritidis can be found in the gastrointestinal tract of humans and other animals, causing diarrhea and other systemic diseases. ${ }^{30}$ Gram-positive bacteria constitute important clinical risk and are responsible for high mortality rates associated with bloodstream infections. In addition, methicillin-resistant Staphylococcus aureus (MRSA) and Enterococcus spp. resistant to vancomycin (VRE) show the importance of the development of new antibiotics. ${ }^{31}$ In this concern, compounds 3a-3f,3k showed selectivity for gram-positive bacteria Staphylococcus aureus and Enterococcus faecalis with MIC values in the range 31.2-62.5 $\mu \mathrm{g} \mathrm{mL} \mathrm{m}^{-1}$. On the other hand, compound $\mathbf{3 j}$ was active against the four tested strains (MIC $31.2 \mu \mathrm{g} \mathrm{mL} \mathrm{L}^{-1}$ ). Compound $\mathbf{3 h}$ also showed discrete inhibition against both gram-positive and gram-negative bacteria. Compound $\mathbf{3 i}$ was the most active, showing inhibitory activity against Staphylococcus aureus (MIC $7.8 \mu \mathrm{g} \mathrm{mL}^{-1}$ ) and Enterococcus faecalis (MIC $7.8 \mu \mathrm{g} \mathrm{mL}^{-1}$ ). These MIC values are lower than those observed for the licensed antibiotic chloramphenicol against $S$. aureus and E. faecalis. This compound also showed moderate inhibition against Salmonella enteritidis (MIC $62.5 \mu \mathrm{g} \mathrm{mL}{ }^{-1}$ ) and Escherichia coli (MIC $62.5 \mu \mathrm{g} \mathrm{mL} \mathrm{m}^{-1}$ ). These data show that bromine at 4-position of the benzene ring is essential for molecule activity. When this atom is shifted to 3-position there is an 8-fold decrease in compound activity against S. aureus and E. faecalis strains. Additionally, the change of bromine by a fluorine atom attached at 4-position produced a molecule 16-fold less active against $S$. aureus and E. faecalis. Electron donating groups at 4-position, like methyl and methoxyl, cause at least a 4-fold decrease in compound activity against $S$. aureus and E. faecalis. These results indicate that compounds $\mathbf{3 h} \mathbf{\mathbf { h }} \mathbf{\mathbf { j }}$ may be used as prototype for new broad-spectrum antibiotics.

\section{Antifungal activity}

The MIC against four Candida type yeasts were determined and reported in Table 3. These Candida species were chosen because they are important opportunistic pathogens besides provide infections due to their virulence factors and resistance to antifungal agents. ${ }^{32}$ Most of amidino pyrazolines did not show high antifungal activity.

Table 2. In vitro antibacterial activity (MIC values)

\begin{tabular}{|c|c|c|c|c|c|}
\hline \multirow[b]{2}{*}{ Compound } & \multicolumn{5}{|c|}{$\mathrm{MIC} /\left(\mu \mathrm{g} \mathrm{mL}^{-1}\right)$} \\
\hline & $\mathrm{Ar}$ & $\begin{array}{l}\text { S. enteritidis } \\
\text { ATCC } 13076\end{array}$ & $\begin{array}{c}\text { E. coli } \\
\text { ATCC } 25922\end{array}$ & $\begin{array}{c}\text { S. aureus } \\
\text { ATCC } 25923\end{array}$ & $\begin{array}{c}\text { E. faecalis } \\
\text { ATCC } 51299\end{array}$ \\
\hline $3 \mathbf{a}$ & $\mathrm{C}_{6} \mathrm{H}_{5}$ & 250 & 250 & 62.5 & 62.5 \\
\hline $3 b$ & $2-\mathrm{MeC}_{6} \mathrm{H}_{4}$ & 250 & 250 & 31.2 & 62.5 \\
\hline $3 c$ & $4-\mathrm{MeC}_{6} \mathrm{H}_{4}$ & 125 & 125 & 31.2 & 62.5 \\
\hline 3d & $2-\mathrm{MeOC}_{6} \mathrm{H}_{4}$ & 250 & 250 & 31.2 & 62.5 \\
\hline $3 e$ & $4-\mathrm{MeOC}_{6} \mathrm{H}_{4}$ & 250 & 250 & 62.5 & 62.5 \\
\hline $3 f$ & 4- $\mathrm{FC}_{6} \mathrm{H}_{4}$ & 125 & 125 & 125 & 125 \\
\hline $3 g$ & 2- $\mathrm{BrC}_{6} \mathrm{H}_{4}$ & 125 & 250 & 250 & 125 \\
\hline $3 \mathbf{h}$ & $3-\mathrm{BrC}_{6} \mathrm{H}_{4}$ & 62.5 & 62.5 & 62.5 & 62.5 \\
\hline $3 \mathbf{i}$ & 4- $\mathrm{BrC}_{6} \mathrm{H}_{4}$ & 62.5 & 62.5 & 7.8 & 7.8 \\
\hline $3 \mathbf{j}$ & 4- $\mathrm{CF}_{3} \mathrm{C}_{6} \mathrm{H}_{4}$ & 31.2 & 31.2 & 31.2 & 31.2 \\
\hline $3 \mathbf{k}$ & $3-\mathrm{NO}_{2} \mathrm{C}_{6} \mathrm{H}_{4}$ & 62.5 & 125 & 31.2 & 31.2 \\
\hline 31 & thiophen-2-yl & 250 & 250 & 125 & 250 \\
\hline $3 m$ & pyridin-2-yl & 1000 & 1000 & 500 & 1000 \\
\hline Chloramphenicol & - & 4 & 4 & 32 & 16 \\
\hline Ampicillin & - & 1 & 4 & 4 & 0.5 \\
\hline
\end{tabular}

MIC: minimum inhibitory concentration. 
Table 3. In vitro antifungal activity (MIC values)

\begin{tabular}{|c|c|c|c|c|c|}
\hline \multirow[b]{2}{*}{ Compound } & \multicolumn{5}{|c|}{$\mathrm{MIC} /\left(\mu \mathrm{g} \mathrm{mL}^{-1}\right)$} \\
\hline & $\mathrm{Ar}$ & $\begin{array}{c}\text { C. krusei } \\
\text { ATCC } 6558\end{array}$ & $\begin{array}{c}\text { C. tropicalis } \\
\text { ATCC } 750\end{array}$ & $\begin{array}{c}\text { C. albicans } \\
\text { ATCC } 90028\end{array}$ & $\begin{array}{l}\text { C. glabrata } \\
\text { ATCC } 2001\end{array}$ \\
\hline $3 \mathbf{a}$ & $\mathrm{C}_{6} \mathrm{H}_{5}$ & 250 & 250 & 250 & 1000 \\
\hline $3 \mathbf{b}$ & $2-\mathrm{MeC}_{6} \mathrm{H}_{4}$ & 125 & 125 & 500 & 500 \\
\hline $3 c$ & $4-\mathrm{MeC}_{6} \mathrm{H}_{4}$ & 31.2 & 62.5 & 125 & 250 \\
\hline 3d & $2-\mathrm{MeOC}_{6} \mathrm{H}_{4}$ & 125 & 125 & 250 & 250 \\
\hline $3 \mathbf{e}$ & 4-MeOC ${ }_{6} \mathrm{H}_{4}$ & 250 & 500 & 500 & 1000 \\
\hline $3 \mathbf{f}$ & $4-\mathrm{FC}_{6} \mathrm{H}_{4}$ & 250 & 250 & 500 & 500 \\
\hline $3 g$ & $2-\mathrm{BrC}_{6} \mathrm{H}_{4}$ & 250 & 250 & 250 & 250 \\
\hline $3 h$ & $3-\mathrm{BrC}_{6} \mathrm{H}_{4}$ & 125 & 125 & 250 & 250 \\
\hline $\mathbf{3 i}$ & 4- $\mathrm{BrC}_{6} \mathrm{H}_{4}$ & 62.5 & 62.5 & 125 & 125 \\
\hline $\mathbf{3 j}$ & $4-\mathrm{CF}_{3} \mathrm{C}_{6} \mathrm{H}_{4}$ & 125 & 125 & 125 & 250 \\
\hline $3 \mathbf{k}$ & $3-\mathrm{NO}_{2} \mathrm{C}_{6} \mathrm{H}_{4}$ & 125 & 62.5 & 125 & 125 \\
\hline 31 & thiophen-2-yl & 500 & 500 & 500 & 500 \\
\hline $3 \mathrm{~m}$ & pyridin-2-yl & - & - & - & - \\
\hline Fluconazole & - & 32 & 2 & 0.5 & 2 \\
\hline Amphotericin B & - & 0.25 & 0.06 & 0.06 & 0.06 \\
\hline
\end{tabular}

MIC: minimum inhibitory concentration.

Only 3c and 3i moderately inhibited the growth of C. krusei and $C$. tropicalis. Compound 3k also showed moderate activity against $C$. tropicalis.

\section{Conclusions}

In summary, a concise and greener method for the synthesis of amidino pyrazolines under sonochemical conditions was achieved. The 13 new compounds were obtained in moderate to good yields without additional purification. The structural characterization was unequivocal using FTIR, ${ }^{1} \mathrm{H}$ and ${ }^{13} \mathrm{C}$ NMR, HRMS and $\mathrm{X}$-ray diffraction. Concerning the antimicrobial tests, some pyrazolines exhibited promising in vitro antibacterial activity. In this sense, the compound $\mathbf{3 i}\left(\mathrm{Ar}=4-\mathrm{BrC}_{6} \mathrm{H}_{4}\right)$ presented the most promising values of MIC, which was comparable with the standard antibiotic chloramphenicol against $S$. aureus and E. faecalis. In this line, future studies can highlight these amidino pyrazoline as drugs prototypes.

\section{Supplementary Information}

Crystallographic data (excluding structure factors) for the structure in this work were deposited in the Cambridge Crystallographic Data Centre as supplementary publication number CCDC 1880378. Copies of the data can be obtained, free of charge, via www.ccdc.cam.ac.uk/conts/ retrieving.html or from the Cambridge Crystallographic Data Centre, CCDC, 12 Union Road, Cambridge CB2 1EZ, UK; fax: +44 1223 336033. E-mail: deposit@ccdc. cam.ac.uk.
Supplementary data are available free of charge at http://jbcs.sbq.org.br as PDF file.

\section{Acknowledgments}

We acknowledge Conselho Nacional de Desenvolvimento Científico e Tecnológico (CNPq, grant 483021/2013-0 for L. Pizzuti) and Fundação de Apoio ao Desenvolvimento do Ensino, Ciência e Tecnologia do Estado de Mato Grosso do Sul (FUNDECT, grant 0180/12 for L. Pizzuti) for financial support. We also thank Coordenação de Aperfeiçoamento de Pessoal de Nível Superior (CAPES) for scholarships to D. Y. Albuquerque and A. C. Damim.

\section{Author Contributions}

Danilo Y. de Albuquerque was responsible for the investigation, methodology and writing original draft; Andressa C. Damim for the investigation and methodology; Eliandro Faoro for the formal analysis, software, validation, visualization, writing-review and editing; Gleison A. Casagrande for the conceptualization, writing-review and editing; Davi F. Back for the investigation, data curation and formal analysis; Sidnei Moura for the investigation, data curation, formal analysis, writing-review and editing; Claudio M. P. de Pereira for the conceptualization and resources; Kelly M. P. de Oliveira for the conceptualization, data curation, methodology, writing-review and editing; Lucas Pizzuti for the conceptualization, data curation, funding acquisition, project administration, writing original draft, writing-review and editing. 


\section{References}

1. Marin, J.; Kumar, R.; J. Enzyme Inhib. Med. Chem. 2014, 29 , 427; da Silva, C. C.; Martins, R.; Lund, R.; Pizzuti, L.; Pereira, C. M. P.; Curr. Bioact. Compd. DOI:10.2174/1573407214666 180730104941.

2. Fiorucci, S.; Meli, R.; Bucci, M.; Cirino, G.; Biochem. Pharmacol. 2001, 62, 1433.

3. Fink, H. A.; Mac Donald, R.; Rutks, I. R.; Nelson, D. B.; Wilt, T. J.; Arch. Intern. Med. 2002, 162, 1349.

4. Christensen, R.; Kristensen, P. K.; Bartels, E. M.; Bliddal, H.; Astrup, A.; Lancet 2007, 370, 1706.

5. Lu, Y.; Wang, G.; Zhong, L.; Zhang, F.; Bai, Q.; Zheng, X.; Lu, Z.; Crop Prot. 2017, 100, 196.

6. Jiang, D.; Zheng, X.; Shao, G.; Ling, Z.; Xu, H.; J. Agric. Food Chem. 2014, 62, 3577.

7. Liu, Y.; Lv, K.; Li, Y.; Nan, Q.; Xu, J.; Sci. Rep. 2018, 8, 1.

8. James, J. P.; Bhat, K. I.; More, U. A.; Joshi, S. D.; Med. Chem. Res. 2018, 27, 546.

9. Oliveira, S.; Pizzuti, L.; Quina, F.; Flores, A.; Lund, R.; Lencina, C.; Pacheco, B. S.; Pereira, C. M. P.; Piva, E.; Molecules 2014, 19, 5806; Ramírez, J.; Rodríguez, M. V.; Quiroga, J.; Abonia, R.; Sortino, M.; Zacchino, S. A.; Insuasty, B.; Arch. Pharm. 2014, 347, 566.

10. Puig-Basagoiti, F.; Tilgner, M.; Forshey, B. M.; Philpott, S. M.; Espina, N. G.; Wentworth, D. E.; Goebel, S. J.; Masters, P. S.; Falgout, B.; Ren, P.; Ferguson, D. M.; Shi, P.-Y.; Antimicrob. Agents Chemother. 2006, 50, 1320.

11. Abdel-Halim, M.; Abadi, A. H.; Engel, M.; Med. Chem. Commun. 2018, 9, 1076.

12. Khalil, N. A.; Ahmed, E. M.; El-Nassan, H. B.; Ahmed, O. K.; Al-Abd, A. M.; Arch. Pharmacal Res. 2012, 35, 995.

13. Li, H.-L.; Su, M.-M.; Xu, Y.-J.; Xu, C.; Yang, Y.-S.; Zhu, H.-L.; Eur. J. Med. Chem. 2018, 155, 725; Zhang, Y.-L.; Qin, Y.-J.; Tang, D.-J.; Yang, M.-R.; Li, B.-Y.; Wang, Y.-T.; Cai, H.-Y.; Wang, B.-Z.; Zhu, H.-L.; ChemMedChem 2016, 11, 1446.

14. Tripathi, A. C.; Upadhyay, S.; Paliwal, S.; Saraf, S. K.; Med. Chem. Res. 2018, 27, 1485.

15. World Health Organization (WHO); WHO Model List of Essential Medicines; 2017, available at https://apps.who.int/ iris/bitstream/handle/10665/273826/EML-20-eng.pdf?ua=1, accessed in September 2019.

16. Huang, T. L.; Eynde, J. J. V.; Mayence, A.; Collins, M. S.; Cushion, M. T.; Rattendi, D.; Londono, I.; Mazumder, L.; Bacchi, C. J.; Yarlett, N.; Bioorg. Med. Chem. Lett. 2009, 19, 5884; Maciejewska, D.; Zabinski, J.; Kazmierczak, P.; Rezler, M.; Krassowska-Swiebocka, B.; Collins, M. S.; Cushion, M. T.; Eur. J. Med. Chem. 2012, 48, 164.
17. Banerjee, B.; Ultrason. Sonochem. 2017, 35, 15.

18. Brasil, S. R.; Capiotto, A. C.; Flores, A. F. C.; Back, D. F.; Faoro, E.; Pizzuti, L.; Orbital: Electron. J. Chem. 2017, 9, 204; dos Santos, E. F. S.; Cury, N. M.; do Nascimento, T. A.; Raminelli, C.; Casagrande, G. A.; Pereira, C. M. P.; Simionatto, E.; Yunes, J. A.; Pizzuti, L.; J. Braz. Chem. Soc. 2017, 28, 217; Pizzuti, L.; Martins, P. L. G.; Ribeiro, B. A.; Quina, F. H.; Pinto, E.; Flores, A. F. C.; Venzke, D.; Pereira, C. M. P.; Ultrason. Sonochem. 2010, 17, 34 .

19. Sheldrick, G. M.; Acta Crystallogr., Sect. A: Found. Adv. 2008 , A64, 112.

20. Farrujia, L. J.; J. Appl. Crystallogr. 1997, 30, 565.

21. Clinical and Laboratory Standards Institute (CLSI); M27-A3: Reference Method for Broth Dilution Antifungal Susceptibility Testing of Yeasts: Approved Standard, $3^{\text {rd }}$ ed.; CSLI: Wayne, 2008; Clinical and Laboratory Standards Institute (CLSI); M07-A9: Methods for Dilution Antimicrobial Susceptibility Tests for Bacteria that Grow Aerobically, $9^{\text {th }}$ ed.; CLSI: Wayne, 2012.

22. Cabrera, M.; Simoens, M.; Falchi, G.; Lavaggi, M. L.; Piro, O. E.; Castellano, E. E.; Vidal, A.; Azqueta, A.; Monge, A.; Ceráin, A. L.; Sagrera, G.; Seoane, G.; Cerecetto, H.; Gonzáles, M.; Bioorg. Med. Chem. 2007, 15, 3356.

23. Světlík, J.; Šallai, L.; J. Heterocycl. Chem. 2002, 39, 363.

24. Koskinen, J. T.; Koskinen, M.; Mutikainen, I.; Tilus, P.; Mannfors, B.; Elo, H.; Z. Naturforsch., B: J. Chem. Sci. 1997, $52,1259$.

25. Bristow, A. W. T.; Webb, K. S.; J. Am. Soc. Mass Spectrom. 2003, 14, 1086; Knolhoff, A. M.; Callahan, J. H.; Croley, T. R.; J. Am. Soc. Mass Spectrom. 2014, 25, 1285.

26. Kéki, S.; Nagy, L.; Török, J.; Tóth, K.; Lévai, A.; Zsuga, M.; Rapid Commun. Mass Spectrom. 2007, 21, 1799.

27. dos Santos, P. R.; Pereira, C. M. P.; Ritter, M.; de Paula, F. R.; Moura, S.; J. Mass Spectrom. 2018, 53, 195.

28. Koskinen, J. T.; Koskinen, M.; Mutikainen, I.; Mannfors, B.; Elo, H.; Z. Naturforsch., B: J. Chem. Sci. 1996, 51, 1771.

29. Bondi, A.; J. Phys. Chem. 1964, 68, 441.

30. Puentes, S. S.; Dunstan, M.; Crit. Care Nurs. Clin. North Am. 2018, 30, 149; Xu, Y.; Tao, S.; Hinkle, N.; Harrison, M.; Chen, J.; Sci. Total Environ. 2018, 616-617, 90.

31. Woodford, N.; Livermore, D. M.; J. Infect. 2009, 59, S4.

32. Wiebusch, L.; de Almeida-Apolonio, A. A.; Rodrigues, L. M. C.; Bicudo, B. P.; Silva, D. B. S.; Lonchiati, D. F.; de Araujo, R. P.; Grisolia, A. B.; de Oliveira, K. M. P.; Asian Pac. J. Trop. Biomed. 2017, 7, 624.

Submitted: July 23, 2019 Published online: October 8, 2019 